\title{
An observational argument against accretion in magnetars
}

\author{
V. Doroshenko ${ }^{1,2}$, A. Santangelo ${ }^{1}$, V. F. Suleimanov ${ }^{1,2,3}$, and S. S. Tsygankov ${ }^{4,2}$ \\ 1 Institut für Astronomie und Astrophysik, Sand 1, 72076 Tübingen, Germany \\ e-mail: doroshv@astro.uni-tuebingen.de \\ 2 Space Research Institute of the Russian Academy of Sciences, Profsoyuznaya Str. 84/32, Moscow 117997, Russia \\ 3 Astronomy Department, Kazan (Volga region) Federal University, Kremlyovskaya str. 18, 420008 Kazan, Russia \\ ${ }^{4}$ Department of Physics and Astronomy, 20014 University of Turku, Finland
}

Received 16 July 2020 / Accepted 18 September 2020

\begin{abstract}
The phenomenology of anomalous X-ray pulsars is usually interpreted within the paradigm of very highly magnetized neutron stars, also known as magnetars. According to this paradigm, the persistent emission of anomalous X-ray pulsars (AXPs) is powered by the decay of the magnetic field. However, an alternative scenario in which the persistent emission is explained through accretion is also discussed in literature. In particular, AXP 4U 0142+61 has been suggested to be either an accreting neutron star or a white dwarf. Here, we rule out this scenario based on the observed X-ray variability properties of the source. We directly compare the observed power spectra of $4 \mathrm{U} 0142+61$ and of two other magnetars, 1RXS J170849.0-400910 and 1E 1841-045 with that of the X-ray pulsar 1A $0535+262$, and of the intermediate polar GK Persei. In addition, we include a bright young radio pulsar PSR B150958 for comparison. We show that, unlike accreting sources, no aperiodic variability within the expected frequency range is observed in the power density spectrum of the magnetars and the radio pulsar. Considering that strong variability is an established feature of all accreting systems from young stellar objects to super-massive black holes and the absence of the variability reports from other magnetars, we conclude that our results also indicate that magnetars, in general, are not powered by accretion.
\end{abstract}

Key words. pulsars: general - stars: magnetars - binaries: general

\section{Introduction}

Magnetars, including soft gamma ray repeaters (SGRs) and anomalous X-ray pulsars (AXPs), are defined as isolated neutron stars (NSs) emitting from radio to X-rays, and they are presumably powered by the dissipation of their very strong magnetic fields $\left(\sim 10^{14} \mathrm{G}\right.$, Thompson et al. 2002; Mereghetti 2008). Such fields are inferred from their rapid spin-down and extreme flaring activity (Katz 1982; Duncan \& Thompson 1992; Thompson et al. 2002). While fields of a similar magnitude have also been suggested for some accreting pulsars (Doroshenko et al. 2010; Reig et al. 2012; Tsygankov et al. 2016), the emission in this case is clearly powered by accretion rather than field decay, so these objects are generally not considered magnetars.

On the other hand, accretion from a fall-back fossil disk, surviving the supernova explosion onto a magnetized NS (van Paradijs et al. 1995; Chatterjee et al. 2000; Alpar 2001), or an isolated white dwarf (WD; Coelho \& Malheiro 2014; Borges et al. 2020) has also been invoked to explain the persistent X-ray emission and spin evolution of AXPs. A serious flaw in this scenario is that it cannot explain the giant flares observed from SGRs, which must still be powered by the decay or reconnection of ultra-strong magnetic fields close to the surface of the NS and triggered by crustal shifts. Furthermore, the timing noise properties of magnetars appear to be consistent with those of radio pulsars but not of accretion-powered sources (Ćerri-Serim et al. 2019). Accretion is thus currently not considered as a mainstream explanation for the AXP phenomenon.

Nevertheless, significant observational efforts aimed at the detection of optical or infrared emission from cool fossil disks, potentially powering accretion, have been undertaken and indeed were successful in revealing the presence of a disk (Hulleman et al. 2000; Kaplan et al. 2001; Ertan \& Çalışkan 2006; Wang et al. 2006; Mereghetti 2008). We note, however, that evidence for the presence of a disk does not necessarily imply that accretion powers the observed emission of AXPs.

To further investigate the accretion scenario, we have taken an alternative and purely phenomenological approach, based on the comparison of the aperiodic variability properties of accreting objects with that of several magnetars including the prototypical AXP 4U 0142+61, often suggested in literature as an accreting system, and of two other bright magnetars, 1RXS J170849.0-400910 and 1E 1841-045. For completeness, we also include a bright radio pulsar PSR B1509-58 for comparison.

If accretion is the mechanism at the base of the observed emission, one shall expect to observe a similar aperiodic variability for accreting sources and magnetars. Accretion is known to be an intrinsically noisy process (Lyubarskii 1997) and all accreting systems, from young stellar objects to active galactic nuclei, do exhibit strong red-noise type aperiodic variability (Rappaport et al. 1971; Oda et al. 1974; Revnivtsev et al. 2009; Scaringi et al. 2015). There is no reason for magnetars to be an exception. In this work, we show, however, that the observed variability properties of AXPs are drastically different from those of accreting systems, and thus conclude that the observed emission is likely not powered by accretion.

\section{Object selection, observations, and analysis}

Being the brightest and arguably the best studied AXPs, 4U $0142+61$ is also the archetypal source discussed in the context of the accretion scenario, and, in fact, one of the two magnetars for 
which substantial observational arguments exist to support this interpretation (the other being 1E 161348-5055 in RCW 103, Esposito et al. 2018). In particular, the detection of mid-infrared emission from a cool disk around the source (Wang et al. 2006) largely motivated the development of the fall-back accretion scenario. The peculiar two-component broadband X-ray spectrum, similar to that of some accretion-powered pulsars (see i.e. Fig. 1 in Doroshenko et al. 2012), has also been interpreted in favor of accretion for this source (see also Ertan et al. 2007; Trümper et al. 2013; Olausen \& Kaspi 2014; Zezas et al. 2015; Borges et al. 2020, and references therein). In addition, its high observed flux and comparatively low spin period $(\sim 8.7 \mathrm{~s})$ make 4U 0142+61 an ideal target for our study.

In this study, we also consider two other magnetars, 1RXS J170849.0-400910 and 1E 1841-045, both of which are bright and have been observed with the same instrument (i.e., NuSTAR), which is also used in this work for $4 \mathrm{U} 0142+61$ and the reference accreting sources. Finally, for completeness, we also include a bright radio pulsar PSR B1509-58, which is also detected in the hard X-ray band and was observed with $\mathrm{NuS}$ TAR (Chen et al. 2016). On the other hand, we have not included $1 \mathrm{E} 161348-5055$ in our sample, since its very long spin period ( $\sim 6.7$ hours) would require very long observations to probe variability on timescales comparable and exceeding the spin period.

Indeed, the power spectral density (PSD) of magnetized NSs and WDs used to describe their variability reveals the presence of strong power-law noise, which is truncated around the spin frequency of the compact object (Revnivtsev et al. 2009). Here, the break is associated with the interaction of the accretion flow with the magnetosphere, which suppresses noise at higher frequencies (Revnivtsev et al. 2009; Suleimanov et al. 2019). If the origin of the observed X-ray emission is the same in accreting pulsars and AXPs, one would expect similar power spectra, that is to say red-type noise with a break around the spin frequency. Considering that the physics of accretion is expected to be the same for similar luminosities, the amplitude of noise relative to the pulsed signal can also be expected to be similar for X-ray pulsars and magnetars. We observe that the lack of secular spinup trends of magnetar candidates would imply, in the context of the fossil-disk accretion scenario, that they are close to corotation with the inner accretion disk regions. This in turn means that the break in the power spectrum can only occur around the spin frequency; additionally, in the accretion scenario, the noise at lower frequencies shall not be suppressed and must be observable. Constraining noise level on timescales of hours is, however, challenging from an observational perspective as it requires very long observations, hence the omission of 1E 161348-5055.

The selection of the reference accreting sources for comparison can be fairly arbitrary as the observed power spectra are qualitatively similar for all accretors (Revnivtsev et al. 2009; Scaringi et al. 2015; Suleimanov et al. 2019). Nevertheless, for a quantitative comparison, we have chosen objects which are as similar as possible to the considered magnetars in terms of phenomenology and the quality of the existing observations. As a reference accreting pulsar, we have selected 1A $0535+262$ observed with NuSTAR in quiescence in Tsygankov et al. (2019a). In this observation, the source was found at a luminosity comparable with that of AXPs and it exhibited a two-component energy spectrum similar to that of X Persei and 4U 0142+61 (Tsygankov et al. 2019a). This is an important point as the hypothesis is such that the persistent emission of AXPs is at least, in part, based on the similarity of their spectra to that of accretion-powered pulsars (Trümper et al. 2013).
Accretion onto a white dwarf has also been suggested to power the persistent emission of AXPs (Coelho \& Malheiro 2014; Borges et al. 2020). As a reference white dwarf accretor, we have chosen the intermediate polar (IP) GK Persei, which was observed by NUSTAR in outburst and thus has one of the highest luminosities among all IPs (Suleimanov et al. 2019). We emphasize, however, that power spectra of all IPs appear to be qualitatively similar (Suleimanov et al. 2019). We stress again that high quality data obtained with the same instrument $(N u S$ $T A R)$ at comparable flux levels are available for all considered objects.

\section{Observed variability in accretors and magnetars}

As already mentioned, all sources in the sample have been observed by NUSTAR. The summary of the observations used in the analysis is presented in Table 1 . To investigate the observed variability properties in all objects, we reduced the data and extracted source light curves in the $3-80 \mathrm{keV}$ energy range, with a time resolution of $0.0625 \mathrm{~s}$ using the HEADAS 6.27 .1 software and current set of calibration files (version 20200526). In each case, the source photons were extracted from a region centered on the source with a radius of $80^{\prime \prime}$. The source signal dominated the count-rate ( $\geq 95 \%$ of all counts in all cases) so the background was not subtracted for a timing analysis. Light curves that were extracted from the two NuSTAR units were corrected to the solar system barycenter and co-added to improve the counting statistics. PSDs were constructed using the powspec task and converted to a format that is readable by XSPEC, as described in Ingram \& Done (2012). They were also rebinned by a constant factor to ensure that at least 20 points contribute to each frequency bin to reduce the statistical bias (Dahlhaus 1988) associated with the Whittle statistics (Whittle 1953) used to fit the resulting PSDs.

To model the PSDs, we chose a broken power law with a break fixed at the spin frequency of a given source (see i.e., Table 1) because all three sources are expected to be close to co-rotation. A zero-width Lorentzian curve with the same frequency and an additional constant were also included in the model to account for pulsations and white noise. The white noise amplitude was fixed to the expected level of two, but it was not subtracted for a clearer presentation of the power spectrum of the AXPs. We also rescaled the frequency axis for plotting so that the break in the PSDs appears at the same location to ease the comparison between individual objects, as was done by Revnivtsev et al. (2009). The results are shown in Fig. 1.

As it is clearly seen from the figure, low-frequency noise dominates the power spectra of the two, well-established accreting objects, 1A $0535+262$ and GK Per. On the contrary, it is completely absent in the PSD of magnetars. To estimate an upper limit on the noise amplitude, we included a broken power law component, fixing the indices $\Gamma_{1,2}$ below and above the break frequency $f_{\text {break }}$ to values similar to that obtained for the other two reference sources, and we calculated the $1 \sigma$ confidence bounds for the amplitudes $A_{\text {noise/pulse }}$ of the noise and pulsed signal using the error command in XSPEC. The results are presented in Table 1.

The noise amplitude in $4 \mathrm{U} 0142+61$ is consistent with zero and, if there is any, it has to be at least by factor of 180 lower than that in the other two objects. In Table 1, we also report the ratio of the pulsed signal amplitude to noise amplitude. Also, in this case, the relative noise power in $4 \mathrm{U} 0142+61$ is substantially lower (by a factor of at least 75) than that of the reference accreting sources. A similar conclusion also holds for the other 
Table 1. Summary of the data used and derived PSD parameters for $4 \mathrm{U} 0142+61,1 \mathrm{~A} 0535+262$, and GK Per.

\begin{tabular}{lcccccc}
\hline \hline Source & 4U 0142+61 & 1A 0535+262 & GK Per & 1RXS J170849.0 & 1E 1841-045 & PSR B1509-58 \\
\hline Obs.id & 30001023003 & 90401370001 & 30101021002 & 30401023002 & 30001025012 & 40024001002 \\
$L_{\mathrm{x}}, 10^{34} \mathrm{erg} \mathrm{s}^{-1}$ & $38.3^{(a)}$ & $6.7^{(b)}$ & $0.14^{(c)}$ & $4.2^{(d)}$ & $0.85^{(d)}$ & $\sim 40^{(e)}$ \\
Exposure, ks & 143 & 55 & 72 & 93 & 100 & 34 \\
Count-rate, s & 3.46 & 2.54 & 1.7 & 1.3 & 1.34 & 1.3 \\
$f_{\text {break }}, \mathrm{Hz}$ & 0.115 & $9.662 \times 10^{-3}$ & $2.849 \times 10^{-3}$ & $9.08676 \times 10^{-2}$ & $8.4825 \times 10^{-2}$ & 6.59 \\
$\Gamma_{1} / \Gamma_{2}$ & $0.7 / 2.0$ & $0.66(6) / 1.89(6)$ & $0.72(6) / 2.30(7)$ & $0.7 / 2.0$ & $0.7 / 2.0$ & $0.7 / 2.0$ \\
$A_{\text {noise }}$ & $\leq 2 \times 10^{-3}$ & $0.44_{-0.07}^{+0.15}$ & $0.37_{-0.01}^{+0.18}$ & $2.7_{-1.8}^{+1.8} \times 10^{-3}$ & $\leq 3 \times 10^{-3}$ & $\leq 4 \times 10^{-4}$ \\
$A_{\text {noise }} / A_{\text {pulse }}$ & $\leq 0.35$ & $40_{-14}^{+22}$ & $220_{-125}^{+730}$ & $\leq 0.2$ & $\leq 0.17$ & $\leq 6^{-4}$ \\
\hline
\end{tabular}

Notes. ${ }^{(a)}$ Using fluxes reported in Weng \& Göğüş (2015) and distance from Borges et al. (2020), ${ }^{(b)}$ Tsygankov et al. (2019a), ${ }^{\left({ }^{c}\right)}$ Suleimanov et al. (2019), ${ }^{(d)}$ Olausen \& Kaspi (2014), ${ }^{(e)}$ Chen et al. (2016). All uncertainties are reported at the $1 \sigma$ confidence level.

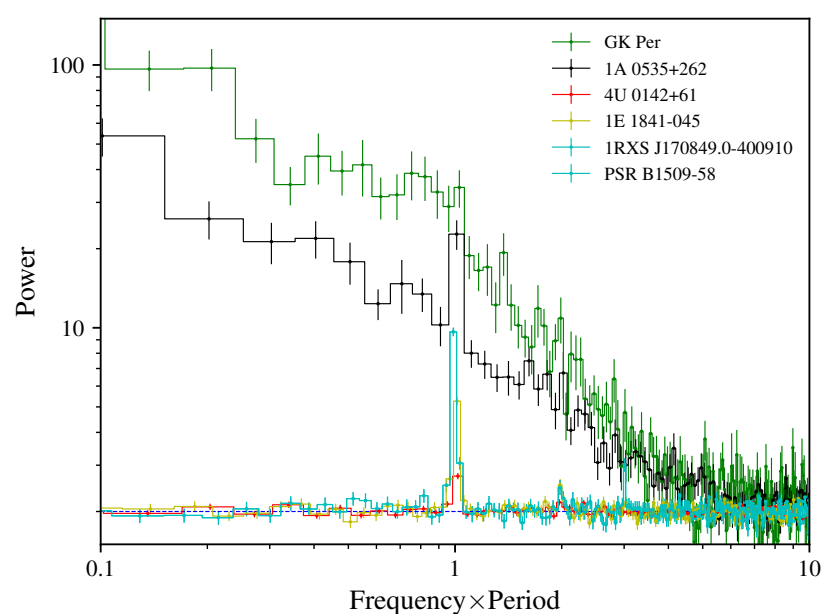

Fig. 1. Power density spectra of the magnetized compact objects discussed in the text and labeled in the legend. The frequency is expressed in units of respective objects spin frequency and power is normalized such that expected white noise level is 2 .

two considered magnetars. We note that, to our best knowledge, there are also no reports of aperiodic variability for the persistent emission in the other magnetars not studied here. Therefore, we conclude that the sources studied in this work do not constitute a special sample, and the absence of any observed aperiodic variability is a strong general argument against the accretionpowered origin of emission from magnetars.

\section{Conclusions}

The recent discovery of the two-component X-ray spectra typical of AXPs in several accreting X-ray pulsars at low luminosity (see Tsygankov et al. 2019a,b) has suggested that such a spectral shape might be a common feature of accretion-powered pulsars at low luminosities. The similarity of the spectra of these objects and AXPs (Doroshenko et al. 2012) can thus be viewed as an argument in favor of the common origin of the observed emission in X-ray pulsars and AXPs, that is to say accretion (Doroshenko et al. 2012; Trümper et al. 2013). We examined, therefore, the hypothesis that the persistent emission of AXP 4U $0142+61$ could indeed be powered by accretion.

We find, however, that, despite the similarity of the observed $\mathrm{X}$-ray spectra, the aperiodic variability that is universally observed in all accreting systems, including the low-luminous $\mathrm{X}$-ray pulsars and accreting white dwarfs (Revnivtsev et al.
2009; Scaringi et al. 2015), is completely absent in 4U 0142+61 RXS J170849.0-400910 and 1E 1841-045. More specifically, this result follows from comparing the observed PSDs of these objects with that of the accreting pulsar 1A 0535+262 (observed in the low luminosity state) and of the intermediate polar GK Persei, which can be considered as representative objects of an accreting neutron star and a white dwarf, respectively. All of these objects were observed with the NUSTAR observatory at a comparable flux and luminosity level, which allowed us to obtain power spectra of a similar quality. We find that despite similar luminosities, counting statistics, and energy spectra, the variability properties of accreting objects and magnetars are drastically different and no evidence for the low-frequency red noise that is typical for accreting sources is detected in the magnetars of the sample. We emphasize that the choice of other reference objects would not alter our conclusions since aperiodic variability is an established feature of accreting systems.

We conclude, therefore, that the observed persistent emission from 4U 0142+61, 1RXS J170849.0-400910, 1E 1841-045, and PSR B1509-58 is not due to accretion, as expected. Considering that $4 \mathrm{U} 0142+61$ is a prime candidate for accretionpowered AXPs and the lack of detected (or reported) variability in any of the magnetar candidates, we conclude that our finding constitutes a strong independent argument against the accretionpowered origin of the persistent X-ray emission in magnetars. This conclusion can be further verified by extending a similar analysis to more sources.

Acknowledgements. V. D. thanks Joachim Trümper for asking the question whether accreting pulsars in low-luminosity state are indeed magnetars. A question which this paper aims to answer. This work was supported by the Russian Science Foundation (Grant 19-12-00423). VFS thanks Deutsche Forschungsgemeinschaft for financial support (Grant DFG-GZ WE 1312/53-1). We thank German Academic Exchange Service (DAAD, project 57405000) and the Academy of Finland (projects 324550, 331951) for travel grants.

\section{References}

Alpar, M. A. 2001, ApJ, 554, 1245

Borges, S. V., Rodrigues, C. V., Coelho, J. G., Malheiro, M., \& Castro, M. 2020, ApJ, 895, 26

Ćerri-Serim, D., Serim, M. M., Şahiner, Ş., Inam, S. ć., \& Baykal, A. 2019, MNRAS, 485, 2

Chatterjee, P., Hernquist, L., \& Narayan, R. 2000, ApJ, 534, 373

Chen, G., An, H., Kaspi, V. M., et al. 2016, ApJ, 817, 93

Coelho, J. G., \& Malheiro, M. 2014, PASJ, 66, 14

Dahlhaus, R. 1988, Ann. Stat., 16, 808

Doroshenko, V., Santangelo, A., Suleimanov, V., et al. 2010, A\&A, 515, A10

Doroshenko, V., Santangelo, A., Kreykenbohm, I., \& Doroshenko, R. 2012, A\&A, 540, L1 
Duncan, R. C., \& Thompson, C. 1992, ApJ, 392, L9

Ertan, Ü. \& Çalışkan, Ş. 2006, ApJ, 649, L87

Ertan, Ü., Erkut, M. H., Eksi, K. Y., \& Alpar, M. A. 2007, ApJ, 657, 441

Esposito, P., Rea, N., \& Israel, G. L. 2018, ArXiv e-prints [arXiv:1803.05716]

Hulleman, F., van Kerkwijk, M. H., \& Kulkarni, S. R. 2000, Nature, 408, 689

Ingram, A., \& Done, C. 2012, MNRAS, 419, 2369

Kaplan, D. L., Kulkarni, S. R., van Kerkwijk, M. H., et al. 2001, ApJ, 556, 399

Katz, J. I. 1982, ApJ, 260, 371

Lyubarskii, Y. E. 1997, MNRAS, 292, 679

Mereghetti, S. 2008, A\&A Rev., 15, 225

Oda, M., Takagishi, K., Matsuoka, M., Miyamoto, S., \& Ogawara, Y. 1974, PASJ, 26, 303

Olausen, S. A., \& Kaspi, V. M. 2014, ApJS, 212, 6

Rappaport, S., Doxsey, R., \& Zaumen, W. 1971, ApJ, 168, L43

Reig, P., Torrejón, J. M., \& Blay, P. 2012, MNRAS, 425, 595

Revnivtsev, M., Churazov, E., Postnov, K., \& Tsygankov, S. 2009, A\&A, 507, 1211
Scaringi, S., Maccarone, T. J., Kording, E., et al. 2015, Sci. Adv., 1, e1500686 Suleimanov, V. F., Doroshenko, V., \& Werner, K. 2019, MNRAS, 482, 3622

Thompson, C., Lyutikov, M., \& Kulkarni, S. R. 2002, ApJ, 574, 332

Trümper, J. E., Dennerl, K., Kylafis, N. D., Ertan, Ü., \& Zezas, A. 2013, ApJ, 764,49

Tsygankov, S. S., Mushtukov, A. A., Suleimanov, V. F., \& Poutanen, J. 2016, MNRAS, 457, 1101

Tsygankov, S. S., Doroshenko, V., Mushtukov, A. e. A., et al. 2019a, MNRAS, 487, L30

Tsygankov, S. S., Rouco Escorial, A., Suleimanov, V. F., et al. 2019b, MNRAS, 483, L144

van Paradijs, J., Taam, R. E., \& van den Heuvel, E. P. J. 1995, A\&A, 299, L41

Wang, Z., Chakrabarty, D., \& Kaplan, D. L. 2006, Nature, 440, 772

Weng, S.-S. \& Göğüss, E., 2015, ApJ, 815, 15

Whittle, P. 1953, Ark. Mat., 2, 423

Zezas, A., Trümper, J. E., \& Kylafis, N. D. 2015, MNRAS, 454, 3366 